\title{
Case Report \\ GNQ-209P Mutation in Metastatic Uveal Melanoma and Treatment Outcome
}

\author{
Nagla Abdel Karim, ${ }^{1}$ Ihab Eldessouki $\left(\mathbb{D},{ }^{1}\right.$ Ahmad Taftaf $\left(\mathbb{D},{ }^{1}\right.$ Deeb Ayham, ${ }^{1}$ Ola Gaber iD, ${ }^{1}$ \\ Abouelmagd Makramalla, ${ }^{2}$ and Zelia M. Correa ${ }^{3}$ \\ ${ }^{1}$ Department of Hematology-Oncology, University of Cincinnati, Cincinnati, OH, USA \\ ${ }^{2}$ Department of Interventional Radiology, University of Cincinnati, Cincinnati, OH, USA \\ ${ }^{3}$ Department of Ophthalmology, University of Cincinnati, Cincinnati, OH, USA
}

Correspondence should be addressed to Ihab Eldessouki; ihab_del@yahoo.com

Received 28 November 2017; Accepted 11 March 2018; Published 4 April 2018

Academic Editor: Raffaele Palmirotta

Copyright (c) 2018 Nagla Abdel Karim et al. This is an open access article distributed under the Creative Commons Attribution License, which permits unrestricted use, distribution, and reproduction in any medium, provided the original work is properly cited.

\begin{abstract}
Metastatic prognosis in uveal melanoma is assessed by gene expression profiling (GEP) testing of the tumor cells, usually obtained by fine needle aspiration (FNA). GEP has demonstrated high accuracy in distinguishing class I and II tumors, both having different metastatic potential. Transcriptomic studies identified distinct mutations including somatic mutations in GNAQ and GNA11, detected in more than $80 \%$, and contribute to the upregulation of the mitogen-activated protein kinase (MAPK) pathway and the development of uveal melanoma (UM). The role of these mutations in treatment selection and possible benefit from targeted therapy are somewhat unclear. However, until the discovery of novel agents, local versus systemic therapies remain options for treatment that can still be considered for disease control in certain cases. We report a series of patients with metastatic UM with distinct mutational profiles. One had significant liver metastases with proven GNQ-209P mutation on tissue biopsy while peripheral blood molecular profiling did not show these mutations. The other three cases had no GNQ-209P mutation. All cases received nab-paclitaxel (Abraxane) as a treatment drug, and we record their responses to treatment and their molecularprofiling results.
\end{abstract}

\section{Introduction}

Uveal melanoma (UM) is significantly less common than cutaneous melanoma and has a distinct molecular pathogenesis. Meanwhile, it is the most common primary intraocular tumor in adults [1]. Despite the high success rate of disease control with local therapy, the potential for developing metastases remains high even after a prolonged period of remission [2-4]. The predominant target organ for metastasis is the liver although disease involvement of the skin, bone, brain, and lungs has also been reported $[5,6]$. Key mutations in the disease are GNAQ and GNA11 mutations. It was reported that $83 \%$ of the cases have somatic mutations in GNAQ or GNA11 [7]. GNAQ gene is the gene coding for the alpha subunit of heterotrimeric $G$ proteins. The latter proteins couple seven-transmembrane domain receptors to intracellular signaling machinery [8], and they are composed of three subunits, namely, alpha, beta, and gamma. The alpha subunit is the G-protein molecular switch, activated when it is bound to guanosine triphosphate (GTP), and when GTP is hydrolyzed to guanosine diphosphate (GDP), it is deactivated [9]. The alpha subunit has a key glutamine that contacts the GTP molecule, located at position 209 (Q209) in G $\alpha \mathrm{q}$ and is substituted when mutated to either leucine or proline [10-13]. At this point, the alpha subunit is locked in a constitutively active state, and its GTPase activity is blocked [14-16]. Taxanes work by preventing microtubule disassembly, so the mitotic functions are inhibited, leading to cell death [17]. They have shown reasonable activity in several phase II studies [18]. Nab-paclitaxel is a solvent-free formula that renders the drug more competent in the treatment of UM. Multiple therapeutic 


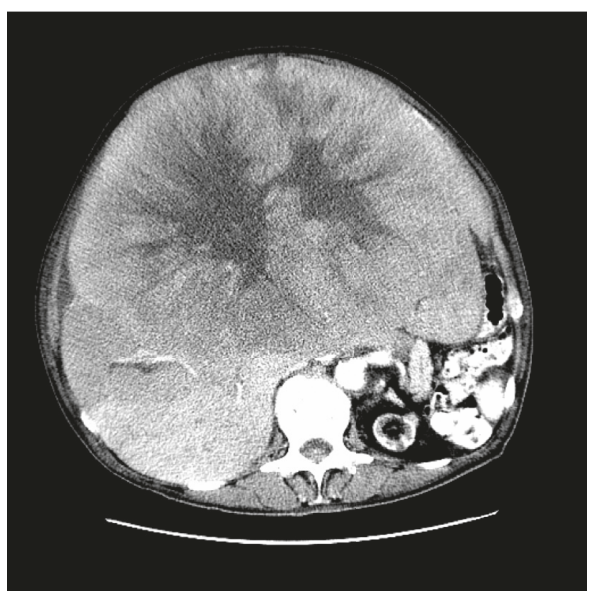

Figure 1: Abdominal CT scan at diagnosis.

approaches for metastatic UM have been studied although none has shown any impact on the overall survival, and thus standard of care has not yet been established for these patients. In our report, we present the case of a patient with metastatic uveal carcinoma to the liver who was successfully treated with nab-paclitaxel, allowing for recovery from lifethreatening spontaneous tumor lysis.

\section{Methods}

GNAQ and GNA11 mutations were assessed by genomic hybridization on paraffin-embedded blocks obtained from the primary tumor tissue. Mutations were followed up by circulating tumor DNA in plasma using next-generation sequencing using serial blood samples.

\section{Case Presentation}

This is a 75-year-old man with a history of choroidal melanoma of the right eye diagnosed in 1984 and treated by radioactive Co-60 plaque. Thirty years later, he presented with progressive abdominal distention, early satiety, and weight loss of 20 pounds over a period of 6 months. He was seen by his primary care physician who requested a CT scan of the abdomen that showed a large hepatic mass measuring $34 \mathrm{~cm}$ by $26 \mathrm{~cm}$, replacing the majority of the liver without retroperitoneal or mesenteric lymphadenopathy (Figure 1). Hepatic tumor biopsy revealed metastatic melanoma consistent with his primary choroidal melanoma. While completing his diagnostic workup, the patient developed generalized weakness prompting his hospital admission due to acute renal failure, hyperkalemia, and spontaneous tumor lysis. He started hemodialysis promptly followed by the administration of weekly nab-paclitaxel $150 \mathrm{mg} / \mathrm{m}^{2}$ and then reduced to $75 \mathrm{mg} / \mathrm{m}^{2}$ thereafter due to severe neutropenia. The patient recovered his renal function as serum creatinine improved from $4.93 \mathrm{mg} / \mathrm{dl}$ to $0.69 \mathrm{mg} / \mathrm{dl}$ (normal values $0.60-1.20 \mathrm{mg} / \mathrm{dl}$ ) and demonstrated clinical improvement of his generalized weakness, abdominal distention, and edema of the legs after three doses of nab-paclitaxel. A repeat abdominal CT scan one month after the therapy revealed

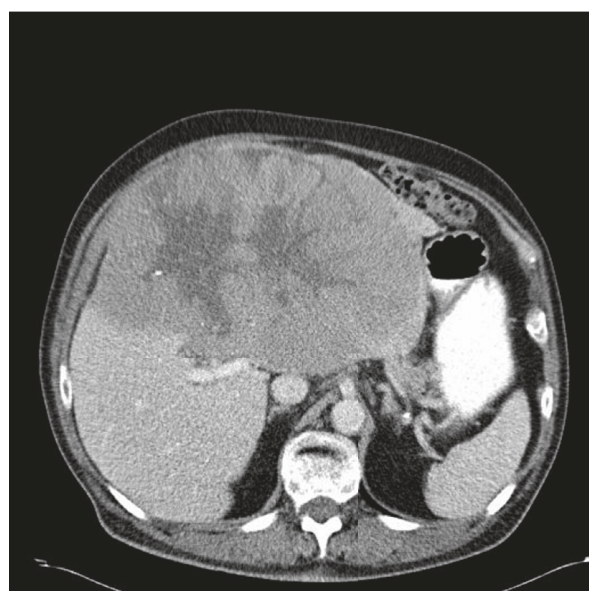

Figure 2: CT scan 24 months after the treatment.

a good response to treatment with significant decrease in tumor burden. This is donated by full clinical recovery and total resolution of tumor lysis manifestations. However, according to RECIST criteria, the response can be minimal followed by maintained stable disease. CT scan of the abdomen after 4 cycles of nab-paclitaxel revealed shrinkage of the hepatic lesion to $24 \times 15 \mathrm{~cm}$ in maximum diameter (approximately $7 \%$ decrease in the largest lesion per RECIST criteria) (Figure 2). This patient is still alive and continues to have excellent functional status, ECOG performance status of I, and no signs or symptoms of disease progression for 32 months now.

Our patient with this metastatic uveal melanoma with extensive liver metastases with GNQ-209P mutation on the tissue biopsy (Figures 3 and 4 ) and undetectable mutations on the peripheral blood molecular profiling in serial follow-up samples suggests marked response to nabpaclitaxel. This can be understood by the dramatic tumor response on CT scans which was accompanied clinically by spontaneous tumor lysis syndrome followed by very prolonged disease control up to 30 months indicating nab-paclitaxel efficacy. All other patients with metastatic ocular melanoma, who did not have the GNQ-209P mutation, did not respond and did not have prolonged survival when treated with nab-paclitaxel.

Our patient has received 8 cycles of Abraxane with initial minimal response followed by no increase and stable tumor size in the following imaging scans. In an attempt to achieve further response, the patient received an anti-PD-L1 in a clinical trial for 9 cycles. No further reduction in tumor size was achieved, and the patient was disqualified from the study after he developed sarcoidosis/interstitial pneumonitis. He was then restarted on Abraxane, achieving clinical and radiological stabilization of his disease with no major toxicities, and remains fully functional. He has received to date 12 cycles of Abraxane (in addition to the prior cycles of Abraxane received initially).

\section{Discussion}

UM has a high potential for developing a rapidly progressive course despite local or systemic therapies [1]. Even with 


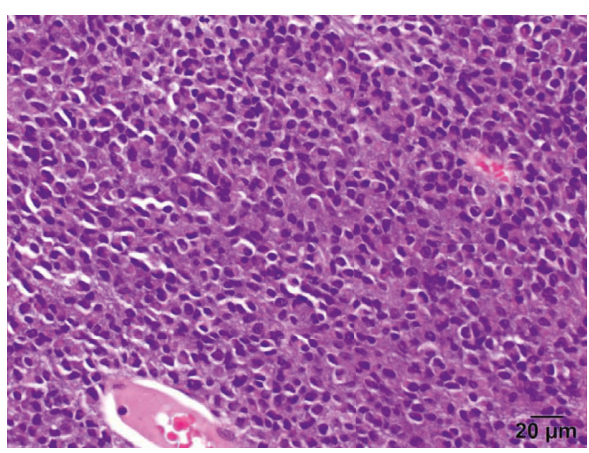

Figure 3: Liver biopsy (H\&E).

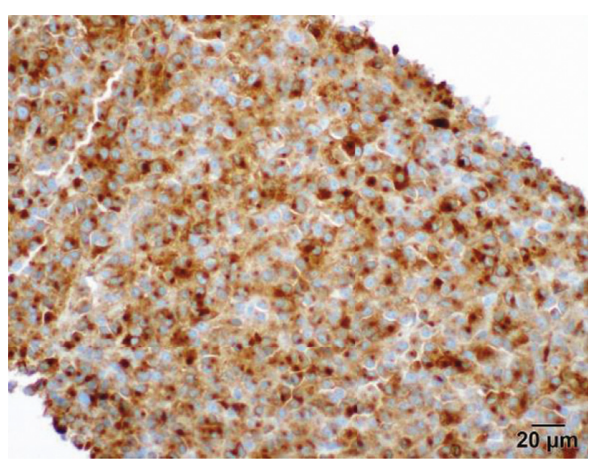

Figure 4: Liver biopsy (MART).

several FDA-approved agents for advanced cutaneous melanoma, there is a lack of agents that show survival benefit in patients with advanced UM. This issue is likely twofold from the rare occurrence of the disease itself as well as a lack of complete understanding regarding the pathogenesis and immunobiology that underlies this disease process. Current studies are ongoing to uncover these uncertainties in hopes of ultimately identifying potentially treatable targets and more effective treatments $[19,20]$. Until a standard of care is established, however, existing treatment options must be applied on a case-by-case basis [21, 22].

In metastatic disease, different approaches have been studied including surgical resection in suitable candidates in addition to local versus systemic infusion of cytotoxic agents. A comprehensive review of the role of metastectomy in selected surgical candidates showed improved survival in patients who had a complete liver metastases resection compared to patients for whom a complete resection was not feasible [23]. Local therapies including the hepatic arterial infusion of melphalan or fotemustine revealed in randomized trial, a significant improvement in progression-free survival (PFS) but not overall survival when compared to the systemic infusion of the same agents [24]. In contrast, a retrospective study from Mayo Clinic showed only improvement in overall survival among patients treated with different local therapies in comparison to different systemic agents including bevacizumab, ipilimumab, and kinase inhibitors [25]. Systemic chemotherapy options have shown minimal benefit in treatment. Single agents such as cisplatin or paclitaxel versus
TABLE 1: Different types of genomic mutations in patients with metastatic melanoma of the liver.

\begin{tabular}{lccc}
\hline $\begin{array}{l}\text { Case } \\
\text { number }\end{array}$ & Age (years) & Gender & Genomic mutation \\
\hline 1 & 75 & Male & GNAQ-Q209P by tissue testing \\
2 & 77 & Female & GNAQ-Q209L by peripheral \\
& 70 & Female & GNACulating DNA \\
3 & 78 & Female & Kit H580y \\
4 & & &
\end{tabular}

TABLE 2: Comparison between GNAQ-Q209P and GNAQ-Q209L.

\begin{tabular}{lcc}
\hline Mutations & $\begin{array}{c}\text { Location of } \\
\text { mutation }\end{array}$ & $\begin{array}{c}\text { Frequency of mutations among } \\
\text { GNAQ-mutated primary uveal } \\
\text { melanoma }\end{array}$ \\
\hline GNAQ-Q209P & Exon 5 & $\sim 64 \%$ \\
GNAQ-Q209L & Exon 5 & $\sim 33 \%$ \\
\hline
\end{tabular}

combined agents such as the BOLD regimen (bleomycin + vincristine + lomustine + dacarbazine) plus recombinant interferon alpha 2-b have been studied with no more than $20 \%$ response rate (RR) and absence of survival advantages $[7,26]$. A similar study to ours that was presented at the ASCO annual meeting shows clinically useful responses in two of four patients with metastatic ocular melanoma treated with nab-paclitaxel [27]. In terms of targeted therapy, the ability to understand the genetic characteristic of UM has helped in identifying different mutations and key signaling pathways that can permit therapeutic intervention at a site specific to the pathway abnormality. UM is genetically characterized by frequent, mutually exclusive mutations in guanine nucleotide-binding protein $\mathrm{G}(\mathrm{q})$ subunit alpha (GNAQ) and guanine nucleotide-binding protein subunit alpha-11 (GNA11) which can be detected in $83 \%$ of patients with UM [12]. GNAQ stimulates the mitogen-activated protein kinase (MAPK), which is parallel to the consequence of mutations in the BRAF or NRAS oncogenes in cutaneous melanomas. Furthermore, GNAQ stimulates the transcriptional coactivator YAP that is essential for UM cell proliferation. The aforementioned MAPK pathway is highly interconnected with the PI3K/ACT pathway, and both of them converge on the same downstream targets. MEK inhibitor, PI3K inhibitor, mTOR inhibitor, and YAP inhibitor each represent novel therapeutic target for UM, and studies are ongoing to uncover the role of these agents either as a single or dual inhibition approach in patients with advanced disease or early disease associated with highrisk features [10, 20, 28].

Our patient presented with acute renal failure secondary to spontaneous tumor lysis, and there was an urgent need for disease control, which was achieved by using systemic chemotherapy with nab-paclitaxel. The absence of standard of care in these patients and the extrapolated data from the phase III trial of nab-paclitaxel when compared to dacarbazine [29] in patients with cutaneous melanoma led to the use of this agent in our patient who was not a candidate for cisplatin. In his case, the tissue from the liver biopsy was insufficient to run 
molecular testing, so we used a liquid biopsy (circulating tumor DNA) obtained from the patient, to search for genomic alterations that came back negative for mutations; however, subsequent liver biopsy revealed GNAQ exon 5 Q209 mutation where the GNA11 mutation or amplification was not detected.

In comparison to this case (Case 1), we had other patients (Case 2, 3, and 4) in our institution, in whom a diagnosis of metastatic UM was made. All patients started on treatment with nab-paclitaxel, but they had metastatic disease that continued to progress. In patients (Cases 2 and 3), a molecular testing of DNA circulating in the blood revealed GNAQ/Q209L mutation while our patient (Case 1) had GNAQ-Q209P (Tables 1 and 2). This might draw our attention that GNQ-209P might be a predictive marker of sensitivity to nab-paclitaxel in metastatic uveal melanoma.

\section{Conclusion}

Although our understanding of the molecular underpinnings of UM continues to improve and certain targeted agents are showing promise, genomic alteration studies might play a role in treatment selection. As we see from the cases we present, GNAQ-Q209P and not Q209L mutation could be associated with a considerable disease control when treated with nabpaclitaxel chemotherapy. We suggest the implication of molecular profiling with specific attention to the status of not only GNAQ but also the exon 209P or Q209L for personalized use of therapies in future clinical trials designed to treat patients with metastatic UM. Such clinical trials are needed to prove the efficacy and survival advantages of nab-paclitaxel in patients with metastatic UM and to study its role in comparison to the evolving targeted or immunotherapeutic agents. In general, reports of rare and less commonly encountered cases can have a pivotal effect on the collective clinical experience and drug research.

\section{Conflicts of Interest}

The authors declare that they have no conflicts of interest.

\section{Acknowledgments}

The authors thank their colleagues in the pathology department who helped with this work.

\section{References}

[1] A. D. Singh, L. Bergman, and S. Seregard, "Uveal melanoma: epidemiologic aspects," Ophthalmology Clinics of North America, vol. 18, no. 1, pp. 75-84, 2005.

[2] J. Scotto, J. F. Fraumeni, and J. A. Lee, "Melanomas of the eye and other noncutaneous sites: epidemiologic aspects," Journal of the National Cancer Institute, vol. 56, no. 3, pp. 489-491, 1976.

[3] E. Kujala, T. Ma"kitie, and T. Kivela", "Very long-term prognosis of patients with malignant uveal melanoma," Investigative Opthalmology and Visual Science, vol. 44, no. 11, p. $4651,2003$.

[4] M. Diener-West, S. M. Reynolds, D. J. Agugliaro et al., "Development of metastatic disease after enrollment in the COMS trials for treatment of choroidal melanoma: collaborative ocular melanoma study group report no. 26," Archives of Ophthalmology, vol. 123, no. 12, pp. 1639-1643, 2005.

[5] The Collaborative Ocular Melanoma Study Group, "Assessment of metastatic disease status at death in 435 patients with large choroidal melanoma in the Collaborative Ocular Melanoma Study (COMS): COMS report no. 15," Archives of Ophthalmology, vol. 119, no. 5, pp. 670-676, 2001.

[6] S. Bakalian, J. C. Marshall, P. Logan et al., "Molecular pathways mediating liver metastasis in patients with uveal melanoma," Clinical Cancer Research, vol. 14, no. 4, pp. 951-956, 2008.

[7] C. D. Van Raamsdonk, K. G. Griewank, M. B. Crosby et al., "Mutations in GNA11 in uveal melanoma," New England Journal of Medicine, vol. 363, no. 23, pp. 2191-2199, 2010.

[8] S. R. Neves, P. T. Ram, and R. Iyengar, "G protein pathways," Science, vol. 296, no. 5573, pp. 1636-1639, 2002.

[9] D. Markby, R. Onrust, and H. Bourne, "Separate GTP binding and GTPase activating domains of a G alpha subunit," Science, vol. 262, no. 5141, pp. 1895-1901, 1993.

[10] C. D. Van Raamsdonk, V. Bezrookove, G. Green et al., "Frequent somatic mutations of GNAQ in uveal melanoma and blue naevi," Nature, vol. 457, no. 7229, pp. 599-602, 2009.

[11] S. Lamba, L. Felicioni, F. Buttitta et al., "Mutational profile of GNAQQ209 in human tumors," PLoS One, vol. 4, no. 8, article e6833, 2009.

[12] M. D. Onken, L. A. Worley, M. D. Long et al., "Oncogenic mutations in GNAQ occur early in uveal melanoma," Investigative Opthalmology and Visual Science, vol. 49, no. 12, pp. 5230-5234, 2008.

[13] J. Bauer, E. Kilic, J. Vaarwater, B. C. Bastian, C. Garbe, and A. De Klein, "Oncogenic GNAQ mutations are not correlated with disease-free survival in uveal melanoma," British Journal of Cancer, vol. 101, no. 5, pp. 813-815, 2009.

[14] C. A. Landis, S. B. Masters, A. Spada, A. M. Pace, H. R. Bourne, and L. Vallar, "GTPase inhibiting mutations activate the alpha chain of Gs and stimulate adenylyl cyclase in human pituitary tumours," Nature, vol. 340, no. 6236, pp. 692-696, 1989.

[15] G. Kalinec, A. J. Nazarali, S. Hermouet, N. Xu, and J. S. Gutkind, "Mutated alpha subunit of the Gq protein induces malignant transformation in NIH 3T3 cells," Molecular and Cellular Biology, vol. 12, no. 10, pp. 4687-4693, 1992.

[16] J. Sondek, D. G. Lambright, J. P. Noel, H. E. Hamm, and P. B. Sigler, "GTPase mechanism of Gproteins from the $1.7-\AA$ crystal structure of transducin a - GDP AIF-4," Nature, vol. 372, no. 6503 , pp. 276-279, 1994.

[17] P. B. Schiff and S. B. Horwitz, "Taxol stabilizes microtubules in mouse fibroblast cells," Proceedings of the National Academy of Sciences, vol. 77, no. 3, pp. 1561-1565, 1980.

[18] R. A. Leon-Ferre and S. N. Markovic, "Nab-paclitaxel in patients with metastatic melanoma," Expert Review of Anticancer Therapy, vol. 15, no. 12, pp. 1371-1377, 2015.

[19] J. J. Luke, P. L. Triozzi, K. C. McKenna et al., "Biology of advanced uveal melanoma and next steps for clinical therapeutics," Pigment Cell and Melanoma Research, vol. 28, no. 2, pp. 135-147, 2015.

[20] S. S. Agarwala, A. M. M. Eggermont, S. O’Day, and J. S. Zager, "Metastatic melanoma to the liver: a contemporary and comprehensive review of surgical, systemic, and regional therapeutic options," Cancer, vol. 120, no. 6, pp. 781-789, 2014.

[21] N. A. Karim, J. Schuster, I. Eldessouki et al., "Pulmonary sarcomatoid carcinoma: University of Cincinnati experience," Oncotarget, vol. 9, no. 3, 2018. 
[22] N. Karim, I. Eldessouki, M. Yellu, T. Namad, J. Wang, and O. Gaber, "A case study in advanced lung cancer patients with vimentin over expression," Clinical Laboratory, vol. 63, no. 10, 2017.

[23] J. F. Pingpank, M. S. Hughes, H. R. Alexander et al., "A phase III random assignment trial comparing percutaneous hepatic perfusion with melphalan (PHP-mel) to standard of care for patients with hepatic metastases from metastatic ocular or cutaneous melanoma," Journal of Clinical Oncology, vol. 28, no. 18 , p. LBA8512, 2010.

[24] J. C. Moser, J. S. Pulido, R. S. Dronca, R. R. McWilliams, S. N. Markovic, and A. S. Mansfield, "The Mayo Clinic experience with the use of kinase inhibitors, ipilimumab, bevacizumab, and local therapies in the treatment of metastatic uveal melanoma," Melanoma Research, vol. 25, no. 1, pp. 59-63, 2015.

[25] L. E. Flaherty, J. M. Unger, P. Y. Liu, W. C. Mertens, and V. K. Sondak, "Metastatic melanoma from intraocular primary tumors," American Journal of Clinical Oncology, vol. 21, no. 6, pp. 568-572, 1998.

[26] T. Kivelä, S. Suciu, J. Hansson et al., "Bleomycin, vincristine, lomustine and dacarbazine (BOLD) in combination with recombinant interferon alpha- $2 b$ for metastatic uveal melanoma," European Journal of Cancer, vol. 39, no. 8, pp. 1115-1120, 2003.

[27] T. J. Smith, S. Temin, E. R. Alesi et al., "American Society of Clinical Oncology provisional clinical opinion: the integration of palliative care into standard oncology care," Journal of Clinical Oncology, vol. 30, no. 8, pp. 880-887, 2012.

[28] E. M. Hersh, M. Del Vecchio, M. P. Brown, and R. F. Kefford, "Phase 3, randomized, open-label, multicenter trial of nabpaclitaxel (nab-P) vs dacarbazine (DTIC) in previously untreated patients with metastatic malignant melanoma (MMM)," Pigment Cell \& Melanoma Research, vol. 25, p. 863, 2012.

[29] P. J. Hesketh, M. G. Kris, E. Basch et al., "Antiemetics: American Society of Clinical Oncology clinical practice guideline update," Journal of Clinical Oncology, vol. 35, no. 28, pp. 3240-3261, 2017. 


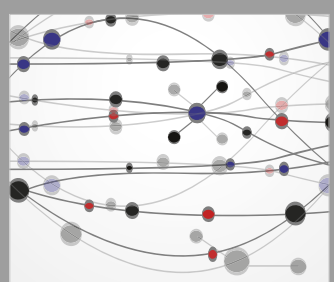

The Scientific World Journal
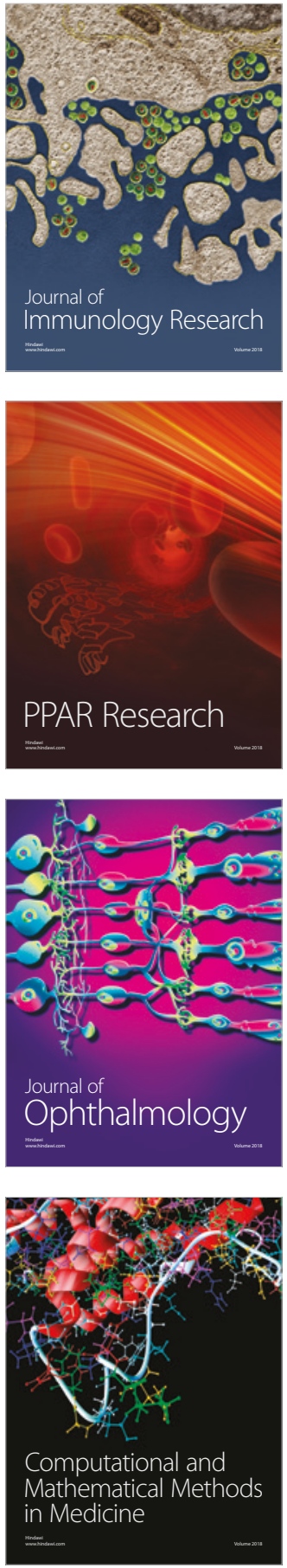

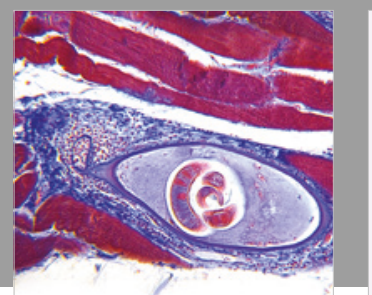

Gastroenterology Research and Practice

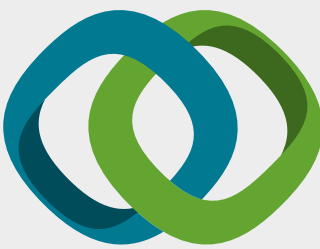

\section{Hindawi}

Submit your manuscripts at

www.hindawi.com
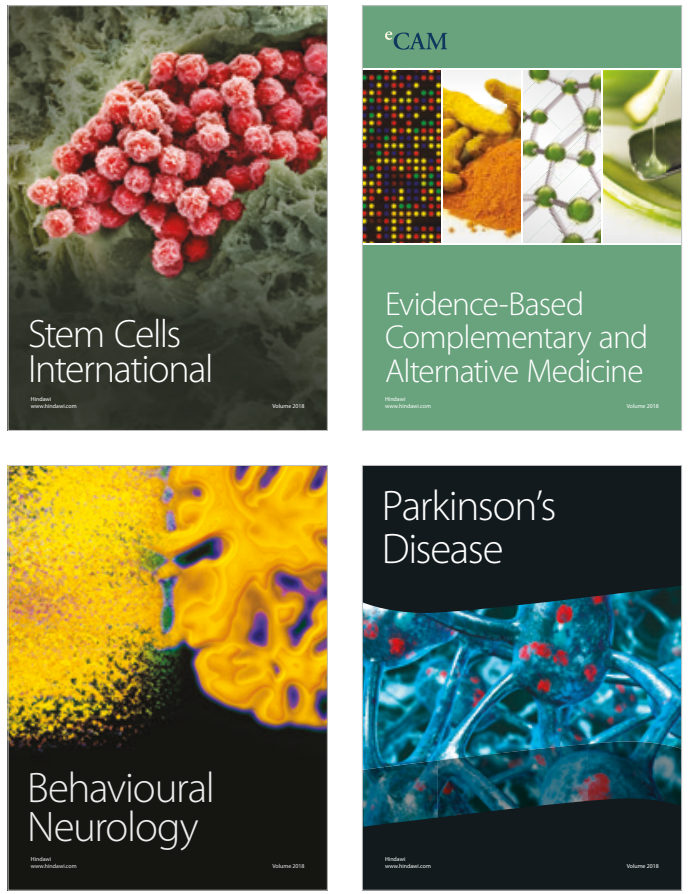

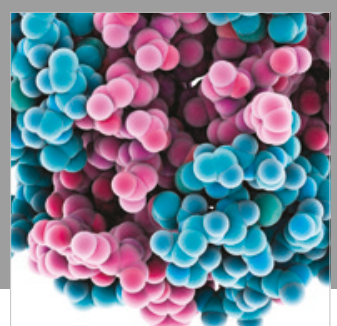

ournal of

Diabetes Research

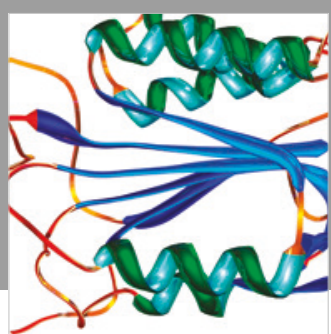

Disease Markers
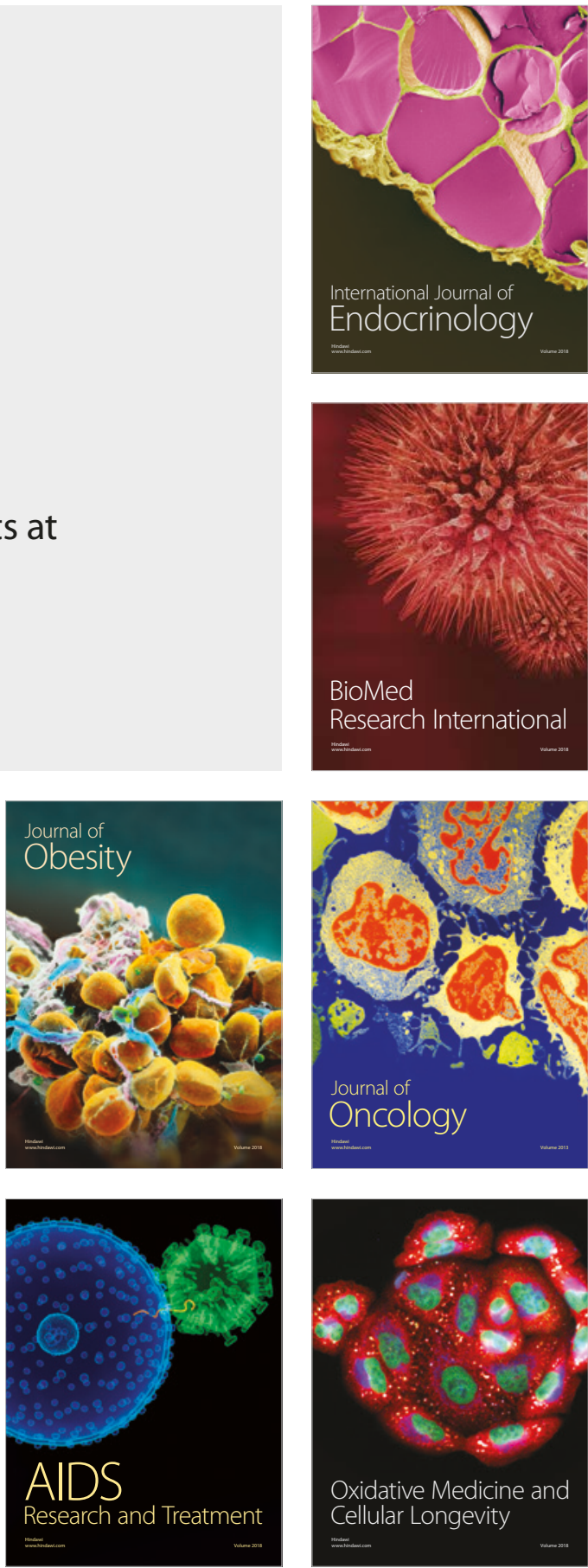